Number of pages: 12

Number of references: 16

Number of Tables: 1

Number of Figures: 6

\title{
Postural stability when walking and exposed to lateral oscillatory motion: benefits from hand supports
}

\author{
Hatice Müjde Ayık and Michael J. Griffin \\ Human Factors Research Unit \\ Institute of Sound and Vibration Research \\ University of Southampton \\ Southampton SO17 1BJ \\ United Kingdom
}

Corresponding author: Michael J. Griffin (M.J.Griffin@soton.ac.uk) 


\begin{abstract}
While walking on a treadmill, 20 subjects experienced lateral oscillations: frequencies from 0.5 to $2 \mathrm{~Hz}$ and velocities from 0.05 to $0.16 \mathrm{~ms}^{-1}$ r.m.s. Postural stability was indicated by ratings of 'discomfort or difficulty in walking', the movement of the centre of pressure beneath the feet, and lateral forces applied to a hand support. Hand support improved postural stability with all frequencies and all velocities of oscillatory motion: the lateral velocity of the centre of pressure reduced by 30 to $50 \%$ when using support throughout motion, by 20 to $30 \%$ when instructed to use the support only when required, and by $15 \%$ during normal walking without oscillation. Improvements in stability, and the forces applied to the hand support, were independent of support height when used continuously throughout motion. When support was used only when required, subjects preferred to hold it 118 to $134 \mathrm{~cm}$ above the surface supporting the feet.
\end{abstract}

\title{
Relevance of the findings for ergonomics practice
}

Lateral oscillation is a main cause of postural instability when standing or walking in transport. A hand-support provides mechanical stabilization and, if held continuously, a hand support provides cues to relative motion that assist the maintenance of postural stability.

Keywords: postural stability; walking; hand support; lateral oscillation; perturbed locomotion. 


\section{Introduction}

Mobile assistive devices (e.g., canes and walking aids) can assist the maintenance of postural stability during quiet standing and when walking. The need for postural assistance can be increased when standing or walking in trains, buses, ships, aircraft and some other environments where human balance is perturbed by the motion of the floor. When walking, and supported on one leg for $80 \%$ of the gait cycle (Woollacott and Tang, 1997), stability might be expected to be less than when standing, so supports may be more beneficial when walking. There are no known studies of how hand supports influence postural stability during perturbed locomotion.

Some assistive devices increase the area at the base of support under the feet and reduce the loading on the lower limbs that provide the reaction forces that counteract the destabilizing effects of body movements (Bateni and Maki, 2005). Similar advantages may be expected for hand supports used in transport. Additionally, light touch contact with a surface, even if it does not provide a force sufficient to stabilize the body, may improve standing stability by providing an additional somatosensory cue to body movement (Jeka and Lackner, 1994, 1995; Tremblay et al., 2004; Clapp and Wing, 1999; Holden et al., 1994). Fingertip contact with a stationary external support (a handrail at a height of $90 \mathrm{~cm}$ ) has been suggested to improve stability during treadmill walking (Dickstein and Laufer, 2004). Apart from any benefits from grasping supports or via light contact, compensatory stepping is used to maintain postural stability when exposed to large perturbations (Maki and Mcllroy, 1997).

With instantaneous increases in horizontal acceleration, standing people have been reported to maintain balance while exposed to accelerations up to $0.76 \mathrm{~ms}^{-2}$ in the backward direction, up to $0.48 \mathrm{~ms}^{-2}$ in the forward direction, and up to $0.33 \mathrm{~ms}^{-2}$ in a sideways direction (Jongkees and Groen, 1942; Graaf and Weperen, 1997). The acceleration in public transport can be greater than these values, so standing people may need to hold supports to maintain stability (Jongkees and Groen, 1942). It has been shown that greater accelerations can be tolerated when using a support (Browning, 1974).

Assuming an inverted pendulum model of the human body, the stabilizing moment from a hand support may be expected to increase as the height of a support increases. A critical handgrip height of $48 \%$ of the body height has been suggested to minimise the muscular force in the arms and trunk when pushing a four-wheeled walker during steady walking (Takanokura, 2010). However, the effects of support height on postural stability during perturbed walking have not been reported.

In transport, motion-induced postural instability is often associated with low frequency oscillations. When exposed to horizontal oscillation, whether a support increases or decreases motion discomfort depends on the frequency and the direction of the oscillation while seated (Wyllie and Griffin, 2007) and while standing (Thuong and Griffin, 2010). The 
discomfort of standing people seems to be increased when a support increases the transmission of high frequency vibration to the upper-body (Thuong and Griffin, 2010).

The experimental study reported here was designed to investigate the effects of hand support, and the height of hand support, on the postural stability of walking subjects perturbed by lateral oscillations at frequencies known to disturb postural stability (Sari and Griffin, 2014). It was hypothesized that 'discomfort or difficulty' in the walking task, and the root-mean-square (r.m.s.) velocity of the lateral centre of pressure would decrease when using a hand support and decrease with increasing height of a hand support.

\section{Methods}

\subsection{Subjects}

Twenty healthy male subjects with median age 28.5 years (range 25 to 40 ), stature $174 \mathrm{~cm}$ (range 166 to 182), mass $70.3 \mathrm{~kg}$ (49 $\mathrm{kg}$ to 88.7 ) participated in the study. All subjects were right-handed. Informed consent was obtained prior to participation in the experiment that was approved by the Human Experimentation Safety and Ethics Committee of the Institute of Sound and Vibration Research.

\subsection{Apparatus}

A treadmill (Kistler Gaitway ${ }^{\circledR}$, Winterthur, Switzerland) incorporating eight force sensors was used to provide the walking task and measure vertical ground reaction forces. Subjects were secured by a loose safety harness that did not restrict walking but would have prevented their knees and hips contacting the floor during a fall, although there were no falls during the study. The treadmill was supported on a six-axis motion simulator in the Human Factors Research Unit at the Institute of Sound and Vibration Research (Fig 1).

\section{FIGURE 1 ABOUT HERE}

A vertically orientated cylindrical handle, rigidly secured to the moving platform of the simulator, was placed $29 \mathrm{~cm}$ to the left of the treadmill to provide a hand support for subjects (Fig. 1). The handle had five differently coloured sections corresponding to the median height of the hip $(92 \mathrm{~cm})$, elbow $(109 \mathrm{~cm})$, thorax $(126 \mathrm{~cm})$, shoulder $(143 \mathrm{~cm})$ and eye $(163 \mathrm{~cm})$ (using anthropometric data for British adults aged 19 to 65 years - Pheasant, 1988). Subjects were discouraged from using the handrail of the treadmill on the right hand side.

Contact forces applied to the hand support in the lateral direction were measured by two single-axis load cells (Tedea Huntleigh Model 1022 1022-20M-C3, Cowbridge, UK), attached at the top and bottom of the vertical handle.

Acceleration in the lateral direction was recorded by accelerometers on the simulator platform (FGP model FA101-A2-5G, Thatcham - Berkshire, UK). Data acquisition via the treadmill software was triggered at the moment the simulator acceleration commenced. The acceleration, vertical ground reaction force, and support contact force data collected by the 
Gaitway ${ }^{\circledR}$ data acquisition system were sampled at 100 samples per second and stored in a personal computer.

\subsection{Experimental Procedure}

While walking on the treadmill, subjects were perturbed by simple transient lateral oscillations - 4.5 cycles of sinusoidal motion modulated by a half sine envelope. The motions were chosen as being broadly representative of the lateral motions experienced in trains (Sari and Griffin, 2014).

Subjects were asked to walk on the treadmill that was set to move at $0.7 \mathrm{~ms}^{-1}$ (a comfortable walking speed) throughout the experiment. This was the average comfortable walking speed preferred by subjects in a preliminary study. While subjects walked on the treadmill, the lateral oscillatory motions were applied at random unpredictable times.

The experiment involved three parts. In Part A and Part B, subjects were exposed to pairs of motion stimuli. The first stimulus was a reference motion $\left(1.0 \mathrm{~ms}^{-2}\right.$ r.m.s. at $\left.1.0 \mathrm{~Hz}\right)$ and was the same throughout the experiment. During the reference motion, subjects held the vertical handle at the median thorax height (S3 position, Fig. 1) continuously throughout the motion. Subjects were asked to report their 'discomfort or difficulty' in walking caused by the second motion (i.e., test motion) relative to the 'discomfort or difficulty' caused by the first motion, assuming the 'discomfort or difficulty' caused by the first motion was 100 .

In Part A (effect of height of support), the test motion was the same as the reference motion. During five of the test motions, subjects held the handle at one of the five vertical positions throughout the motion. During one test motion, they did not hold the handle (i.e., without support).

In Part B (effect of frequency and magnitude of motion), at each of seven frequencies (0.5, $0.63,0.8,1.0,1.25,1.6,2.0 \mathrm{~Hz})$, the motions were presented at the velocity of $0.16 \mathrm{~ms}^{-1}$ r.m.s., corresponding to seven acceleration magnitudes $(0.5,0.63,0.8,1.0,1.25,1.6$ and 2.0 $\mathrm{ms}^{-2}$ r.m.s.). At $1 \mathrm{~Hz}$, the motions were also presented at six velocities $(0.05,0.064,0.08,0.1$, 0.125 and $0.16 \mathrm{~ms}^{-1}$ r.m.s.), corresponding to six acceleration magnitudes $(0.315,0.4,0.5$, $0.63,0.8$ and $1.0 \mathrm{~ms}^{-2}$ r.m.s.). These test motions were applied in two conditions: with support (subjects held the support at the S3 position throughout the motion) and without support.

In Part C (preferred support height), there was no reference motion. Subjects were exposed to oscillations at seven different frequencies $(0.5,0.63,0.8,1,1.25,1.6,2.0 \mathrm{~Hz})$ with a velocity of $0.16 \mathrm{~ms}^{-1}$ r.m.s. They were invited to hold the support when required during exposure to the oscillation. They were free to hold the support at whatever position they preferred so as to stabilize their body against the motion. The preferred holding position was recorded by the experimenter.

Parts $A, B$, and $C$ were applied in sequence in a single trial but the test motions within each part were presented in an independent random order for each subject. 
Gait measure (i.e., centre of pressure) and the lateral force applied to the hand support were also gathered with subjects walking normally without oscillation, both with and without holding the support at a height of $126 \mathrm{~cm}$.

\subsection{Analysis}

The force time-histories (from eight force sensors in the treadmill) were processed to determine the centre of pressure (COP) time histories during each motion. The COP is the point that identifies the location of the total ground reaction forces acting under both feet. The COP velocity in the lateral direction was obtained by differentiating the lateral COP position after filtering the centre of pressure position using a low-pass Bessel filter with an 8- $\mathrm{Hz}$ cut-off frequency.

The change in postural stability when holding the support was quantified by percentage reductions in the subjective measure (i.e., 'discomfort or difficulty' ratings) and the objective measure (i.e., r.m.s. COP velocity calculated over the period from the start to the end of each lateral oscillation):

$$
\text { Reduction }(\%)=\frac{\text { measure evaluated with support }- \text { measure evaluated without support }}{\text { measure evaluated without support }} * 100
$$

The lateral force applied by the hand was obtained from the sum of the forces measured by the load cells at the top and bottom of the vertical handle. Mass cancelation was performed in the time domain by subtracting the product of the acceleration and the mass of the handle from the total measured force.

An example of the COP position of a subject exposed to $0.8-\mathrm{Hz}$ lateral oscillation at $0.8 \mathrm{~ms}^{-2}$ r.m.s. is shown in Fig. 2. The COP position shows the location of the ground reaction forces in the lateral direction and is indicative of lateral foot placement. The COP velocity indicates the rate of change of COP position (Fig. 2). An example of the lateral force applied to the vertical handle is shown in Fig. 2c.

\section{FIGURE 2 ABOUT HERE}

Non-parametric statistical tests were performed using SPSS (version 17). Friedman analysis of variance was used to test for differences between multiple conditions and the Wilcoxon matched-pairs signed ranks test was used to investigate differences between pairs of conditions. Associations between variables were investigated using Spearman's rank correlation.

\section{Results}

\subsection{Effect of support during perturbed walking (part $A$ and part $B$ ) and normal walking}

When walking without perturbation (i.e., no oscillation), holding the support at the median thorax height reduced the r.m.s. COP velocity by $15.6 \%$ ( $p<0.01$, Wilcoxon, Fig. $3 b)$. When 
walking with perturbation, with any frequency of motion (Fig. 4b) and with any magnitude of motion (Fig. 5b), the use of the hand support resulted in a greater percentage reduction in r.m.s. COP velocity than during unperturbed walking ( $p<0.01$, Wilcoxon).

The r.m.s. force applied to the support during unperturbed walking was less than the r.m.s. force during lateral oscillation with any height of the hand support (Fig. $3 \mathrm{c}$ ), any frequency of motion (Fig. 4c), and any magnitude of motion (Fig. $5 c$ ) ( $p<0.01$, Wilcoxon).

\section{FIGURES 3, 4 AND 5 ABOUT HERE}

\subsection{Preferred height for hand support (part $C$ )}

In Part $\mathrm{C}$ of the experiment, subjects held the support when required and at whatever position they preferred during exposure to $0.16 \mathrm{~ms}^{-1}$ r.m.s. at all frequencies from 0.5 to $2.0 \mathrm{~Hz}$. About $60 \%$ of subjects chose to hold the support at the median thorax height (118 to $134 \mathrm{~cm}$ above the surface supporting the feet, Fig. 6). The preferred support height was independent of the frequency of oscillation ( $p=0.09$, Friedman).

\section{FIGURE 6 ABOUT HERE}

\subsection{Effect of height of hand support (part A)}

During $1-\mathrm{Hz}$ lateral oscillation at $1.0 \mathrm{~ms}^{-2}$ r.m.s., the 'discomfort or difficulty' ratings did not depend on the height at which the subjects held the hand support ( $p=0.224$, Friedman). However, as may be expected, the 'discomfort or difficulty' rating was greater without the support than with any of the support heights ( $p<0.01$, Wilcoxon; Fig. 3a).

The r.m.s. COP velocity and the r.m.s. lateral force applied to the hand support were also independent of support height ( $p=0.78$ and $p=0.06$, respectively, Friedman; Fig. $3 b$ and Fig. 3c), but the r.m.s. COP velocity was greater without support than with any of the five support heights ( $p<0.01$, Wilcoxon; Fig. 3b).

\subsection{Effect of frequency of oscillation (part B)}

With a velocity of $0.16 \mathrm{~ms}^{-1}$ r.m.s. at all frequencies, the 'discomfort or difficulty' in walking was independent on the frequency of oscillation when using the hand support ( $p=0.098$, Friedman; Fig. 4a) but dependent on the frequency of oscillation when not using the support $(p<0.01$; Friedman, Fig. 4a). When not using the support, 'discomfort or difficulty' in walking during exposure to $0.5 \mathrm{~Hz}$ oscillation was significantly greater than during $1-\mathrm{Hz}$ and $1.25-\mathrm{Hz}$ oscillations ( $p<0.05$, Wilcoxon). The 'discomfort or difficulty' ratings during exposure to $1-\mathrm{Hz}$ motion were significantly less than those during exposure to $1.6-\mathrm{Hz}$ and $2-\mathrm{Hz}$ oscillation ( $p<0.01$, Wilcoxon). At all frequencies, the 'discomfort or difficulty' was less when the support was held throughout the oscillation than when it was not held ( $p<0.01$, Wilcoxon; Fig. 4a).

At all frequencies, the r.m.s. COP velocity was less when the support was held throughout oscillation, or the support was held only when required, than when it was not held $(p<0.05$, Wilcoxon; Fig. 4b). When the support was used if required, the r.m.s. COP velocity during 1- 
$\mathrm{Hz}, 1.25-\mathrm{Hz}$ and $2-\mathrm{Hz}$ was significantly greater than when the support was used throughout the motion at the same frequencies ( $p<0.01$ at $1 \mathrm{~Hz}$ and $2 \mathrm{~Hz}, p<0.05$ at $1.25 \mathrm{~Hz}$; Wilcoxon). The variability in r.m.s. COP velocity (expressed in terms of inter-quartile range) was between $6 \mathrm{~cm} . \mathrm{s}^{-1}$ (at $0.5 \mathrm{~Hz}$ ) and $31.42 \mathrm{~cm} . \mathrm{s}^{-1}$ (at $1.6 \mathrm{~Hz}$ ) without support, between $14.08 \mathrm{~cm} . \mathrm{s}^{-1}$ (at 0.5 $\mathrm{Hz}$ ) and $22.53 \mathrm{~cm} \cdot \mathrm{s}^{-1}$ (at $0.63 \mathrm{~Hz}$ ) with support held throughout the oscillation, and between $10.94 \mathrm{~cm} . \mathrm{s}^{-1}$ (at $1.25 \mathrm{~Hz}$ ) and $22 \mathrm{~cm} \cdot \mathrm{s}^{-1}$ (at $1.0 \mathrm{~Hz}$ ) with the support held if required (Fig. 4b).

With the same motion velocity at all frequencies, the r.m.s. COP velocity was independent of the frequency of oscillation, both with support and without support ( $p>0.05$, Friedman; Fig. 4b).

The lateral r.m.s. force applied by the hand during oscillation at $0.16 \mathrm{~ms}^{-1}$ r.m.s. was dependent on the frequency of oscillation, both when used throughout oscillation and when used only when required ( $p<0.01$, Friedman; Fig. $4 c)$. The lateral r.m.s. force applied to the hand support when used throughout the $0.5-\mathrm{Hz}$ oscillation was significantly greater than during exposure to $1-\mathrm{Hz}$ oscillation ( $p=0.01$, Wilcoxon). When the support was used if required, the lateral r.m.s. force applied to the hand support during exposure to $0.5-\mathrm{Hz}$ oscillation was significantly greater than during exposure to $1.25-\mathrm{Hz}$ lateral oscillation $(p<0.05$, Wilcoxon). At all frequencies, when holding the support only when required, the force was greater than when holding the support continuously throughout the oscillation $(p<0.01$, Wilcoxon; Fig. 4c), except at the three highest frequencies (i.e., 1.25, 1.6, and $2 \mathrm{~Hz}$ ). The variability in r.m.s. force applied to the hand support (expressed in terms of inter-quartile range) was between $7.34 \mathrm{~N}$ (at $0.5 \mathrm{~Hz}$ ) and $22.62 \mathrm{~N}$ (at $1.6 \mathrm{~Hz}$ ) with the support held throughout the oscillation, and between $11.05 \mathrm{~N}($ at $1.25 \mathrm{~Hz}$ ) and $32.81 \mathrm{~N}($ at $2.0 \mathrm{~Hz}$ ) with the support held if required (Fig. 4b).

When using the support continuously throughout the motion, the percentage reductions in the 'discomfort or difficulty' ratings depended on the frequency of oscillation $(p<0.01$, Friedman; Table 1). The percentage reduction in 'discomfort or difficulty ratings' from using the hand support at $0.5 \mathrm{~Hz}$ was significantly greater than during $1-\mathrm{Hz}$ and $1.25-\mathrm{Hz}$ oscillation $(p<0.01$, Wilcoxon, Table 1). The percentage reductions in 'discomfort or difficulty ratings' from using the hand support during exposure to $1-\mathrm{Hz}$ and $1.25-\mathrm{Hz}$ lateral oscillation were significantly smaller than during exposure to $2-\mathrm{Hz}$ oscillation ( $\mathrm{p}<0.05$, Wilcoxon, Table 1$)$. The percentage reductions in the r.m.s. COP velocity were independent of the frequency of oscillation, both when the hand support was used throughout the oscillation and when used only when required ( $p=0.089$ and $p=0.922$, respectively; Friedman). The median percentage reduction in r.m.s. COP velocity was greater when the support was held throughout the oscillation than when used only when required $(p<0.01$, Wilcoxon, Table 1$)$.

TABLE 1 ABOUT HERE

\subsection{Effect of magnitude of oscillation (part B)}


When exposed to lateral oscillation at $1 \mathrm{~Hz}$, the 'discomfort or difficulty' ratings increased with increasing magnitude of oscillation, both with and without the hand support $(p<0.01 ; r=0.973$ with support, $r=0.928$ without support ,Spearman; Fig. 5a). At all magnitudes of oscillation, the 'discomfort or difficulty' ratings were less when a support was used ( $p<0.01$, Wilcoxon).

At each magnitude of oscillation, the r.m.s. COP velocity was less when the support was held throughout the motion than when walking without support ( $p<0.01$, Wilcoxon; Fig. $5 b)$. The r.m.s. COP velocity increased with increasing magnitude of oscillation without support $(p<0.01, r=0.858$, Spearman, Fig. $5 b)$ but was independent of the magnitude of oscillation when holding the support ( $p=0.056$; Friedman, Fig. $5 b)$. The lateral r.m.s. force applied to the hand support also tended to increase with increasing magnitude of oscillation ( $p=0.021$, $r=0.893$, Spearman, Fig. $5 c$ ).

\subsection{Discussion}

The results show that with all magnitudes and all frequencies of lateral oscillation, holding the hand support improved postural stability when perturbed by lateral oscillations, as indicated by decreased 'discomfort or difficulty' ratings and decreased COP velocity (Fig. 4 and Fig. 5).

Forces less than $1 \mathrm{~N}$ applied by a fingertip contact to a stationary support have been reported to improve stability during quiet standing (Jeka and Lackner, 1994, 1995; Clapp and Wing 1999) and during normal (i.e., unperturbed) walking (Dickstein and Laufer, 2004). During normal walking without oscillation in the current study, lateral forces on the hand support were around $6.9 \mathrm{~N}$ r.m.s. and are similar to the mean of $5 \mathrm{~N}$ applied by standing subjects to a stationary support via fingertip contact (Jeka and Lackner, 1994). Slightly higher forces are consistent with a full grasp of the vertical handle and the different postural requirements when walking. During oscillation, the forces were in the range 14 to $29 \mathrm{~N}$ r.m.s. (35 to $83 \mathrm{~N}$ peak) when the support was used throughout the motion and in the range 24 to $38 \mathrm{~N} \mathrm{r.m.s.}(67$ to $118 \mathrm{~N}$ peak) when the support was used only when required (Fig. 4c and Fig. 5c). The support improved stability (i.e., reduced COP velocity) more during perturbed walking than during normal walking, consistent with the perturbation increasing the risk of fall and requiring greater forces, and more rapidly applied forces, to counteract the destabilizing effects of lateral oscillation.

'Discomfort and difficulty' ratings reported by walking subjects in this experiment are an indication of perceived risk of fall and can be used to quantify how much the walking stability was improved by holding a hand support during perturbed walking (Table 1). Holding the support reduced 'discomfort or difficulty' ratings with all frequencies $(0.5$ to $2 \mathrm{~Hz})$ and with all magnitudes of oscillation ( 0.315 to $1.0 \mathrm{~ms}^{-2}$ r.m.s.) (Fig. 4a and Fig. 5a). With a similar support and moderate magnitudes of lateral sinusoidal oscillation (at $0.5,1.0$ and $2.0 \mathrm{~Hz}$ ), holding a bar had little effect on the comfort of standing people (Thuong and Griffin, 2010), possibly because postural instability was not the main source of discomfort when standing at the magnitudes and frequencies used in that study. 
The 'discomfort or difficulty' ratings obtained using relative magnitude estimation in the current study were dependent on the frequency of oscillation when not holding the support, even though the motion velocity was maintained at $0.16 \mathrm{~ms}^{-1}$ r.m.s. at each frequency (Fig. 4a). In a previous study with the same motion waveform and a range of motion magnitudes, with a velocity of $0.16 \mathrm{~ms}^{-1}$ r.m.s. there was a $90 \%$ self-rated probability of losing balance, independent of the frequency of oscillation between 0.5 and $2 \mathrm{~Hz}$ (Sari and Griffin, 2014). The significant reduction in 'discomfort and difficulty' ratings at $1.0 \mathrm{~Hz}$ and $1.25 \mathrm{~Hz}$ in the present study may have been caused by subjects synchronizing their stepping with the lateral oscillation. This is consistent with reduced force applied by the hand at $1 \mathrm{~Hz}$, both when a support was used throughout the oscillation and when a support was used only if required. When motions are severe, the scale previously used for reporting the perceived probability of losing balance (Sari and Griffin, 2014) appears to become less sensitive due to saturation (towards the maximum possible value of $100 \%$ ). The measure of 'discomfort or difficulty' obtained using magnitude estimation in the current study seems to have been more sensitive to factors influencing walking stability (e.g., the frequency of oscillation and the use of supports).

With the same motion velocity at each frequency, the COP velocities when holding and when not holding the hand support in the current study were independent of the frequency of oscillation (Fig. 4b), consistent with the previous study without hand support (Sari and Griffin, 2014). The lowest and the highest frequencies (i.e., 0.5 and $2 \mathrm{~Hz}$ ) produced greater 'discomfort or difficulty' ratings, and holding the hand support appeared most beneficial at these frequencies (Fig 4a, Table 1), but the percentage improvement in COP velocity from holding the hand support was independent of the frequency of oscillation (Table 1). The lateral r.m.s. force applied to the hand support was greatest at the lowest frequency (Fig. 4c), probably because with the same velocity at each frequency the displacements were greatest at the lowest frequency and the larger low frequency displacement provoked greater voluntary action from subjects to counteract the destabilizing effects of the oscillation. Using a 'grasping strategy' to recover from the destabilising effects of perturbation may have reduced 'stepping strategies', as seen in the reduced COP velocity at all frequencies (Fig $4 b$ ).

When subjects held the support continuously throughout oscillation, the 'discomfort or difficulty' ratings, the COP velocities, and the lateral forces applied to the hand support were similar with all support heights (Fig. 3). If the support was purely providing a force needed for mechanical stabilization of the body, the subjective and objective evaluations of postural instability may be expected to decrease with increasing support height, due to the increased balancing moment provided by support contact forces with a greater moment arm. The absence of an effect of support height suggests the support may not have only provided mechanical stabilization but also a sensory cue that assisted spatial orientation (Jeka and Lackner, 1994; Jeka, 1997). 
Although similar benefit was obtained with all heights of hand support, when subjects grasped the support only when it was required, they mostly preferred to hold it 118 to $134 \mathrm{~cm}$ above the surface supporting the feet, which may have been the most comfortable height for most subjects. The preferred height may be expected to depend on the stature and the reach of subjects. The findings reported here were obtained with fit adult males (statures in the range 166 to $182 \mathrm{~cm}$ ). A lower preferred height can be expected for shorter people, including children, and a greater influence of motion on instability may be expected with some members of the travelling public. The effect of a support on walking stability may also be expected to depend on the direction of oscillation (Thuong and Griffin, 2012), the waveform of the perturbation (Thuong and Griffin, 2011) and the physical characteristics of the subjects.

The COP velocity was less when using the hand support, either continuously or only when required, than when not using the support (Fig. 4b). The percentage reductions in COP velocity were greater, but the forces applied to the hand support were generally reduced, when holding the support throughout oscillation than when grasping it only when required (Table 1, Fig. 4c). Postural stability during perturbed walking is therefore more threatened when supports are used only if required than when supports are used continuously throughout the perturbation. If a support is held only when required it only provides mechanical stabilization. If a support is held continuously it also provides cues to relative motion between the body and the environment, both during perturbed walking and during unperturbed walking (Jeka and Lackner, 1994; Jeka, 1997). It may be concluded that the extent to which holding a support provides stabilizing reaction forces or useful sensory cues depends on both the perturbation and whether the support is held continuously or only when required.

\section{Acknowledgements}

This study was conducted with the support of Sociéte Nationale des Chemins de fer Français (SNCF), Paris, France. The assistance of Cedric Gallais is gratefully acknowledged.

\section{References}

Bateni, H. and Maki, B.E., 2005. Review Article: Assistive Devices for Balance and Mobility: Benefits, Demands, and Adverse Consequences. Arch Phys Med Rehabil., 86, 134-145.

Browning, A.C., 1974. The tolerance of the general public to a speed differential between adjacent floors with special reference to pedestrian conveyors: Exploratory and preliminary experiments, Tech. Report 74076, Farnborough, England, Royal Aircraft Establishment.

Clapp, S. and Wing, A.M., 1999. Light touch contribution to balance in normal bipedal stance. Exp Brain Res. 125m 521-524.

Dickstein, R. and Laufer, Y., 2004. Light touch and center of mass stability during treadmill locomotion. Gait and Posture, 20, 41-47. 
Holden, M., Ventura, J. and Lackner, J.R., 1994. Stabilization of posture by precision contact of the index finger. Journal of Vestibular Research, 4, 285-301.

Jeka, J.J. and Lackner, J.R., 1994. Fingertip contact influences human postural control. Exp Brain Res, 100, 495-502.

Jeka, J.J. and Lackner, J.R., 1995. The role of haptic cues from rough and slippery surfaces in human postural control. Exp Brain Res, 103, 267-276.

Jeka, J.J., 1997. Light Touch Contact as a Balance Aid. Physical Therapy, 77 (5), 476-487.

Jongkees, L.B.W. and Groen, J.J., 1942. De standvastigheid van de mens [The stability of the human body]. Nederlands Tiidschrift voor Geneeskunde 86, 1401-1407.

Graaf, B. De and Van Weperen, W., 1997. The retention of balance: An explanatory study into the limits of acceleration without losing equilibrium. Human Factors and Ergonomics Society, 39(1), 111-118.

Maki, B.E. and Mcllroy, W.E., 1997. The role of limb movements in maintaining upright stance: the "change-in-support" strategy Phys Ther. 77(5):488-507.

Pheasant, S., 1988. Bodyspace: anthropometry, ergonomics and design. Taylor \& Francis, London.

Sari, H.M. and Griffin, M.J., 2014. Postural stability when walking: Effect of the frequency and magnitude of lateral oscillatory motion. Applied Ergonomics, 45(2): 293-299.

Takanokura M., 2010. Optimal handgrip height of four-wheeled walker on various road conditions to reduce muscular load for elderly users with steady walking. J.Biomech., 43,843848.

Tremblay, F., Mireault, A-C., Dessureault, L., Manning, H. and Sveistrup, H., 2004. Postural stabilization from fingertip contact: I. Variations in sway attenuation, perceived stability and contact forces with aging. Exp. Brain Research, 157, 275-285.

Thuong, O. and Griffin, M.J., 2010. The vibration discomfort of standing persons: the effect of body supports. Proc. IMechE, 224, Part F: J. Rail and Rapid Transit, JRRT369.

Thuong, O. and Griffin, M.J., 2011. The vibration discomfort of standing persons: evaluation of random and transient motions. Ergonomics, 54, 1228-1239.

Thuong, O. and Griffin, M.J., 2012. The vibration discomfort of standing persons: Relative importance of fore-and-aft, lateral, and vertical vibration. Applied Ergonomics, 43, 902-908.

Woollacott, M.H. and Tang, P., 1997. Balance control during walking in the older adult: Research and its implications. Physical Therapy 77, 646-660.

Wyllie, I.H. and Griffin, M.J., 2007. Discomfort from sinusoidal oscillation in the roll and lateral axes at frequencies between 0.2 and $1.6 \mathrm{~Hz}$. The Journal of the Acoustical Society of America 121, 2644-2654. 
Postural stability when walking and exposed to lateral oscillatory motion: benefits from hand supports Ayik, H. M. \& Griffin, M. J. 2015 In : Ergonomics. 58, 2, p. 291-300. 


\section{FIGURE CAPTIONS}

Fig. 1. Experimental apparatus.

Fig. 2. Example time histories of the centre of pressure (COP) and lateral force applied to the hand support for a subject walking while exposed to $0.8 \mathrm{~ms}^{-2}$ r.m.s. lateral oscillation at $0.8 \mathrm{~Hz}$ : (a) COP position in the lateral direction; (b) COP velocity in the lateral direction; (c) lateral force applied to the hand support: — support held only when required during oscillation, - - - - support held continuously throughout the oscillation, ........... support held continuously without oscillation.

Fig. 3. Effects of support height while exposed to $1.0 \mathrm{~ms}^{-2}$ r.m.s. lateral oscillation at $1.0 \mathrm{~Hz}$ (medians and inter-quartile ranges): (a) 'discomfort or difficulty' ratings; (b) r.m.s. COP velocity during oscillation and during normal walking (without oscillation); (c) lateral r.m.s. force on the hand support during oscillation and during normal walking (without oscillation):

$\boldsymbol{\Delta}$ support held throughout the oscillation (support height of $126 \mathrm{~cm}$ ), without support.

Fig. 4. Effects of frequency while exposed to $0.16 \mathrm{~ms}^{-1}$ r.m.s. lateral oscillation (medians): (a) 'discomfort or difficulty' ratings. (b) r.m.s. COP velocity during oscillation and during normal walking (without oscillation). (c) lateral r.m.s. force applied to the hand support during oscillation and during normal walking (without oscillation): $\boldsymbol{\Delta}$ support held throughout the oscillation (support height of $126 \mathrm{~cm}$ ), $\mathbf{a}$ support held only when required, without support.

Fig. 5. Effects of motion magnitude while exposed to lateral oscillation at $1 \mathrm{~Hz}$ (medians and inter-quartile ranges): (a) 'discomfort or difficulty' ratings. (b) r.m.s. COP velocity during oscillation and during normal walking (without oscillation). (d) lateral r.m.s. force applied to the hand support during oscillation and during normal walking (without oscillation): support held continuously throughout oscillation (support height of $126 \mathrm{~cm}$ ),, $\mathbf{a}$ support used only when required, $\bullet$ without support.

Fig. 6. Percentage of subjects preferring each support height at each frequency of oscillation with a velocity of $0.16 \mathrm{~ms}^{-1}$ r.m.s. 
Table 1 Median percentage reductions in 'discomfort or difficulty' ratings and r.m.s. COP velocity from holding the hand support throughout oscillation and only if required, as a function of the frequency of $0.16 \mathrm{~ms}^{-1}$ r.m.s. lateral oscillation.

\begin{tabular}{|c|c|c|c|}
\hline \multirow{2}{*}{$\begin{array}{c}\text { Frequency } \\
\text { (Hz) }\end{array}$} & $\begin{array}{c}\text { Percentage reductions in } \\
\text { 'discomfort or difficulty' } \\
\text { rating (\%) }\end{array}$ & \multicolumn{2}{|c|}{$\begin{array}{c}\text { Percentage reductions in r.m.s. COP } \\
\text { velocity (\%) }\end{array}$} \\
\cline { 2 - 4 } & $\begin{array}{c}\text { Support used throughout } \\
\text { oscillation }\end{array}$ & $\begin{array}{c}\text { Support used } \\
\text { throughout oscillation }\end{array}$ & $\begin{array}{c}\text { Support used if } \\
\text { required }\end{array}$ \\
\hline 0.5 & 45 & 43 & 33 \\
\hline 0.63 & 44 & 37 & 22 \\
\hline 0.8 & 33 & 43 & 29 \\
\hline 1.0 & 22 & 47 & 21 \\
\hline 1.25 & 20 & 45 & 28 \\
\hline 1.6 & 31 & 34 & 22 \\
\hline 2.0 & 45 & 51 & 20 \\
\hline
\end{tabular}




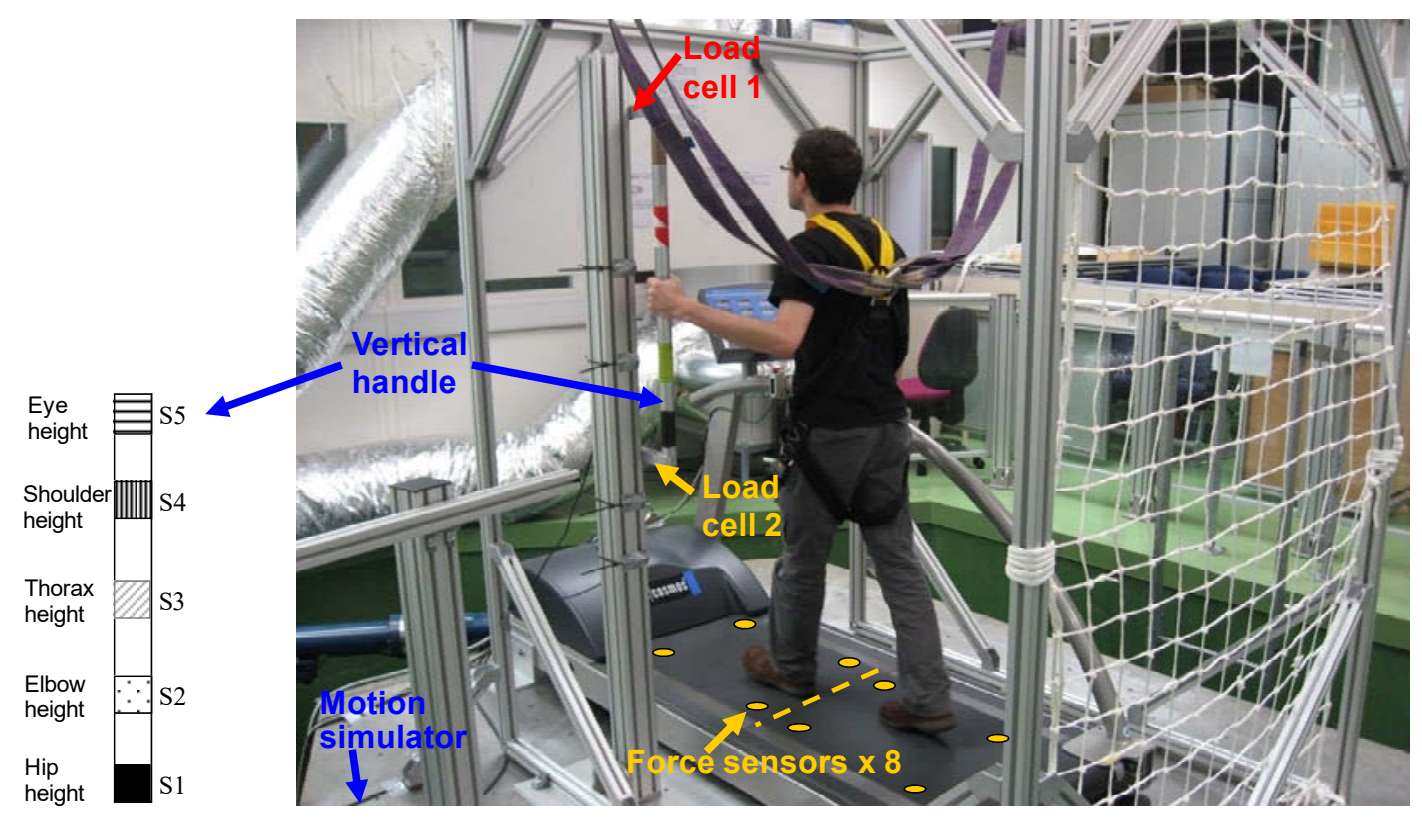

Fig. 1. Experimental apparatus. 

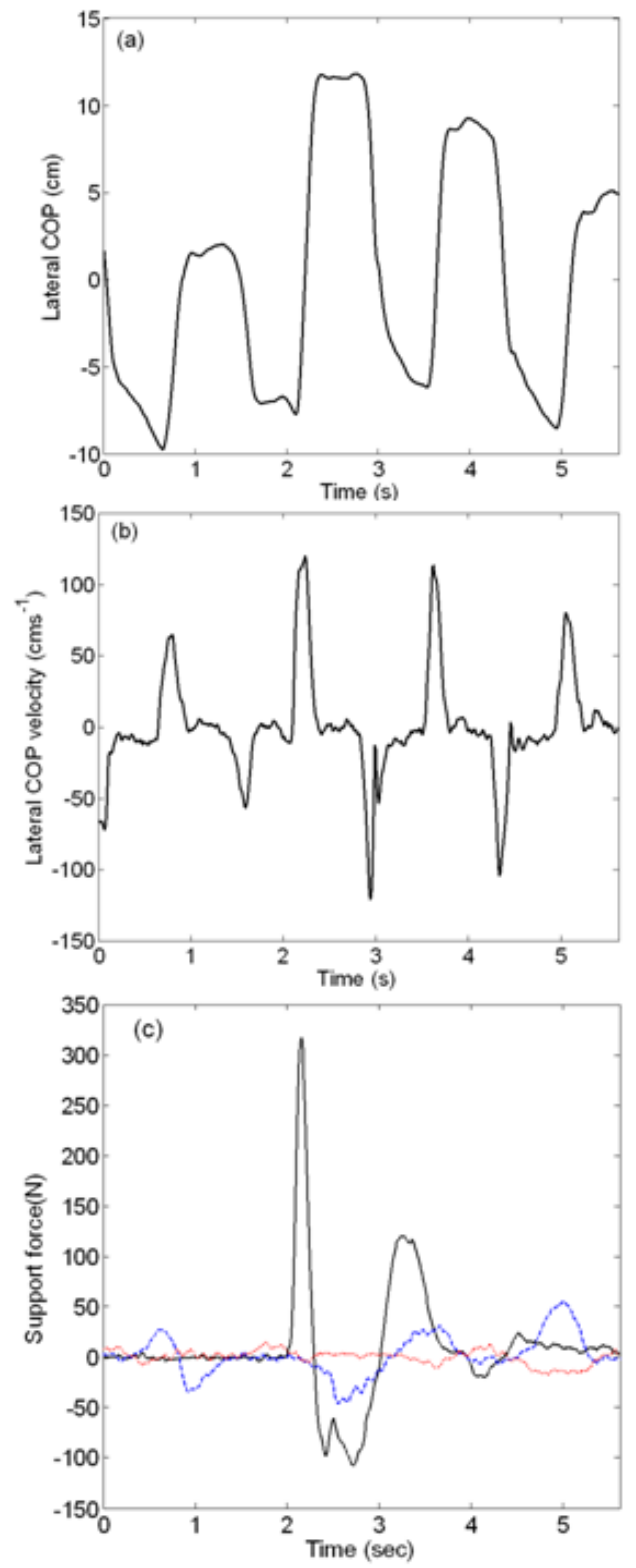

Fig. 2: Example time histories of the centre of pressure (COP) and lateral force applied to the hand support for a subject walking while exposed to $0.8 \mathrm{~ms}^{-2}$ r.m.s. lateral oscillation at 0.8 $\mathrm{Hz}$ : (a) COP position in the lateral direction; (b) COP velocity in the lateral direction; (c) lateral force applied to the hand support: support held if required during oscillation, support held continuously throughout the oscillation, support held continuously without oscillation. 

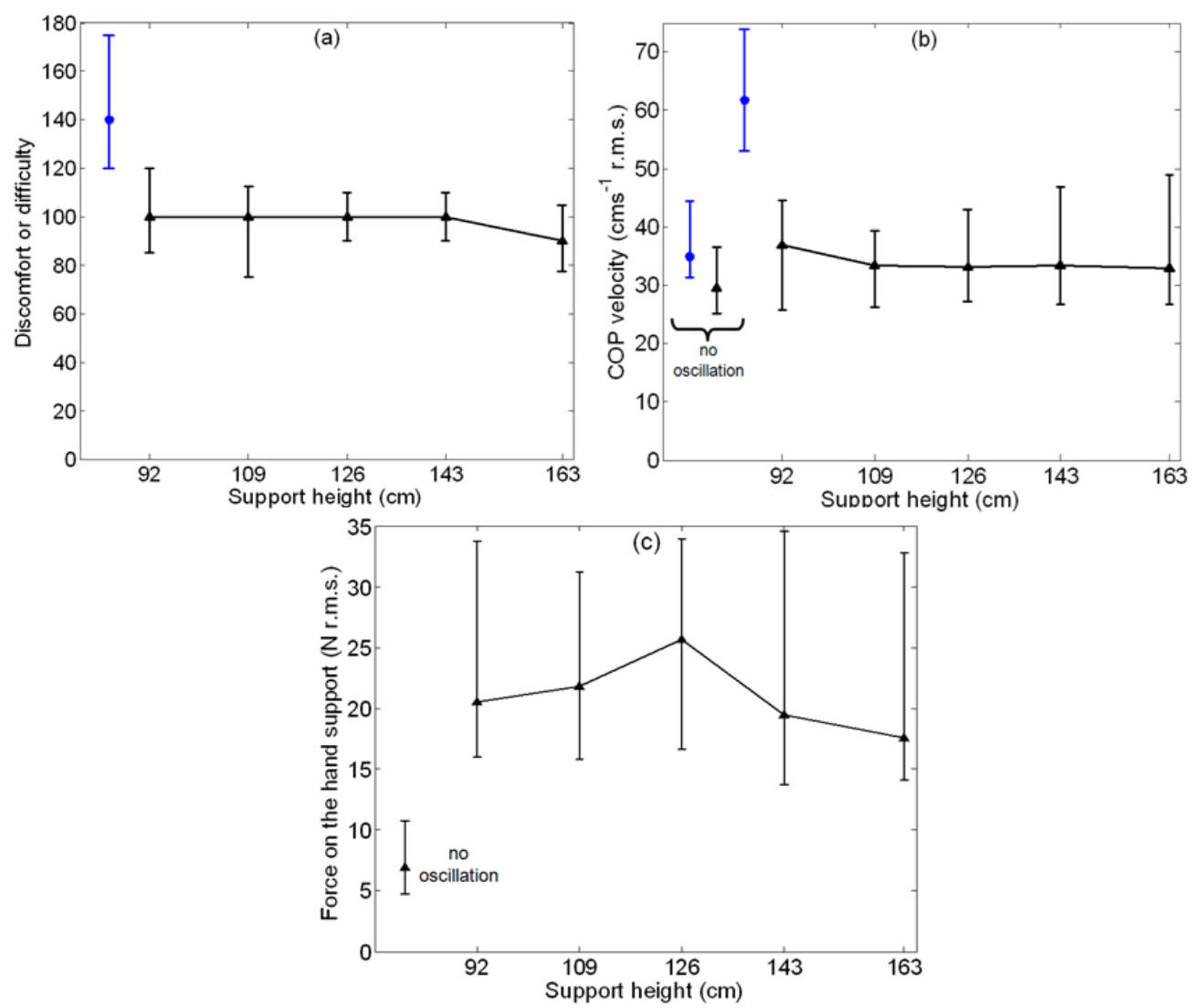

Fig. 3: Effects of support height while exposed to $1.0 \mathrm{~ms}^{-2}$ r.m.s. lateral oscillation at $1.0 \mathrm{~Hz}$ (medians and inter-quartile ranges): (a) 'discomfort or difficulty' ratings; (b) r.m.s. COP velocity during oscillation and during normal walking (without oscillation); (c) lateral r.m.s. force on the hand support during oscillation and during normal walking (without oscillation):

A support held throughout the oscillation (support height of $126 \mathrm{~cm}$ ), without support. 

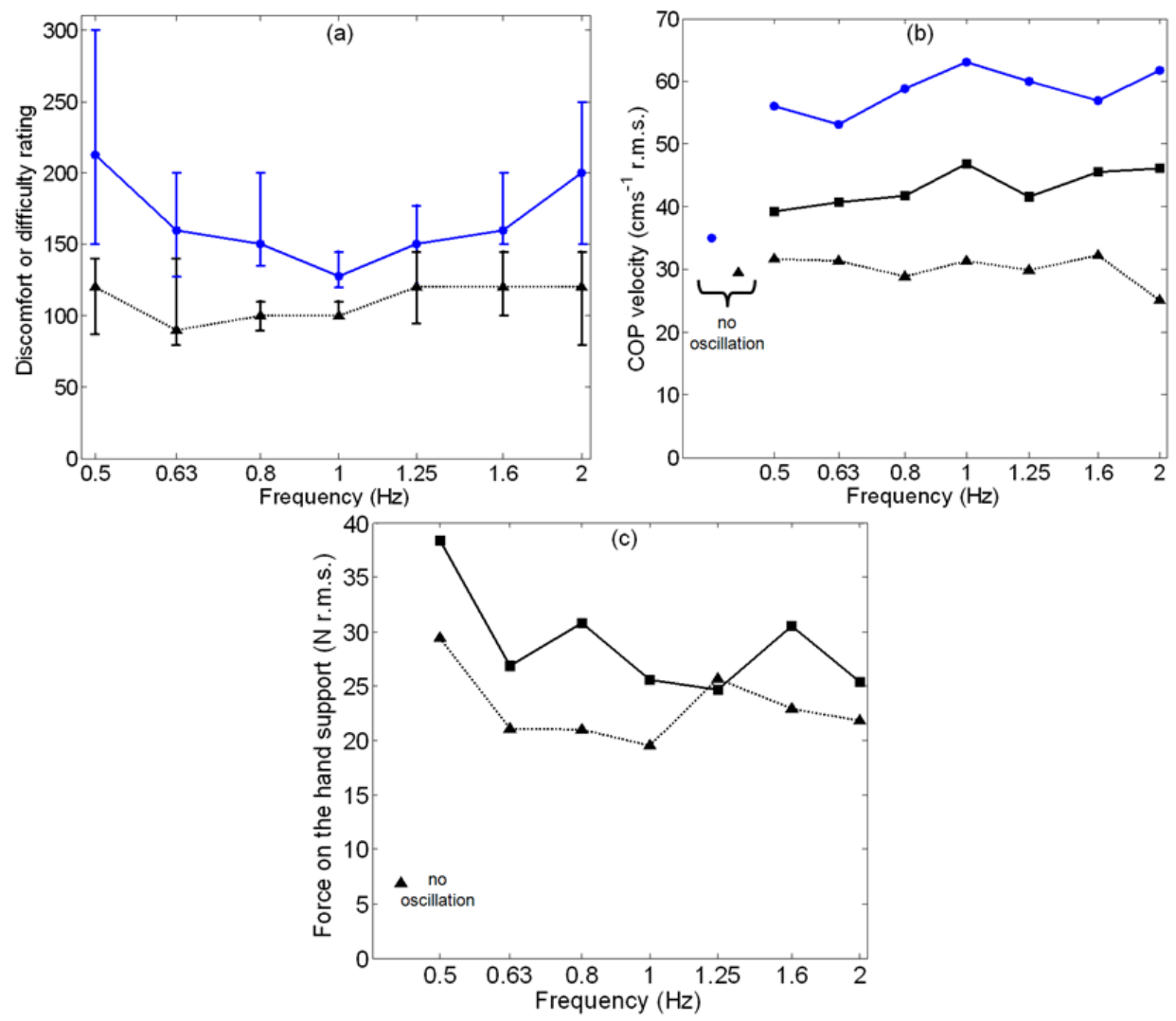

Fig. 4: Effects of frequency while exposed to $0.16 \mathrm{~ms}^{-1}$ r.m.s. lateral oscillation (medians): (a) 'discomfort or difficulty' ratings. (b) r.m.s. COP velocity during oscillation and during normal walking (without oscillation). (c) lateral r.m.s. force applied to the hand support during oscillation and during normal walking (without oscillation): $\boldsymbol{\Delta}$ support held throughout the oscillation (support height of $126 \mathrm{~cm}$ ), $\mathbf{a}$ support held only if required, without support. 

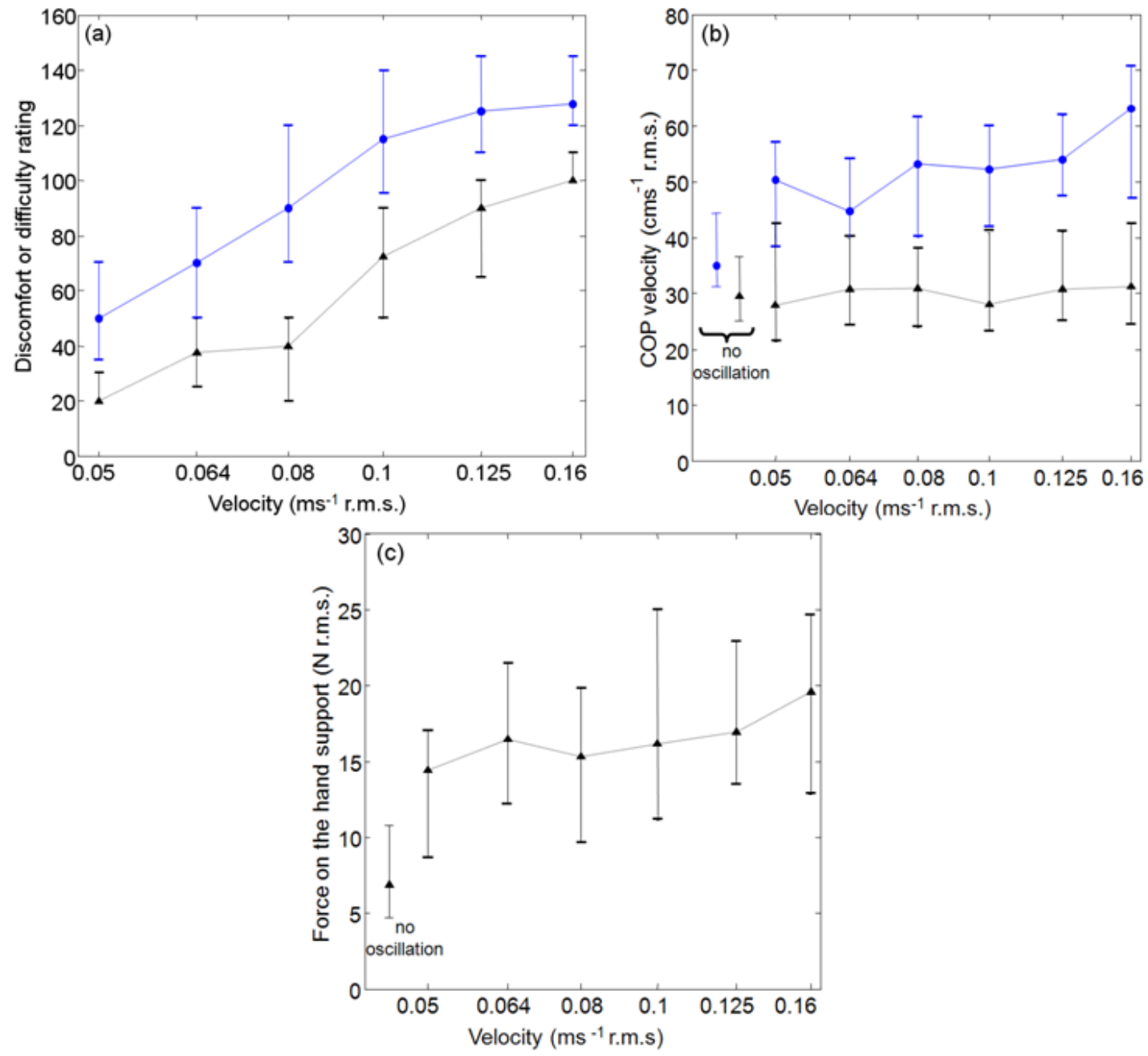

Fig. 5: Effects of motion magnitude while exposed to lateral oscillation at $1 \mathrm{~Hz}$ (medians and inter-quartile ranges): (a) 'discomfort or difficulty' ratings. (b) r.m.s. COP velocity during oscillation and during normal walking (without oscillation). (d) lateral r.m.s. force applied to the hand support during oscillation and during normal walking (without oscillation): $\boldsymbol{\Delta}$ support held continuously throughout oscillation (support height of $126 \mathrm{~cm}$ ), without support. 


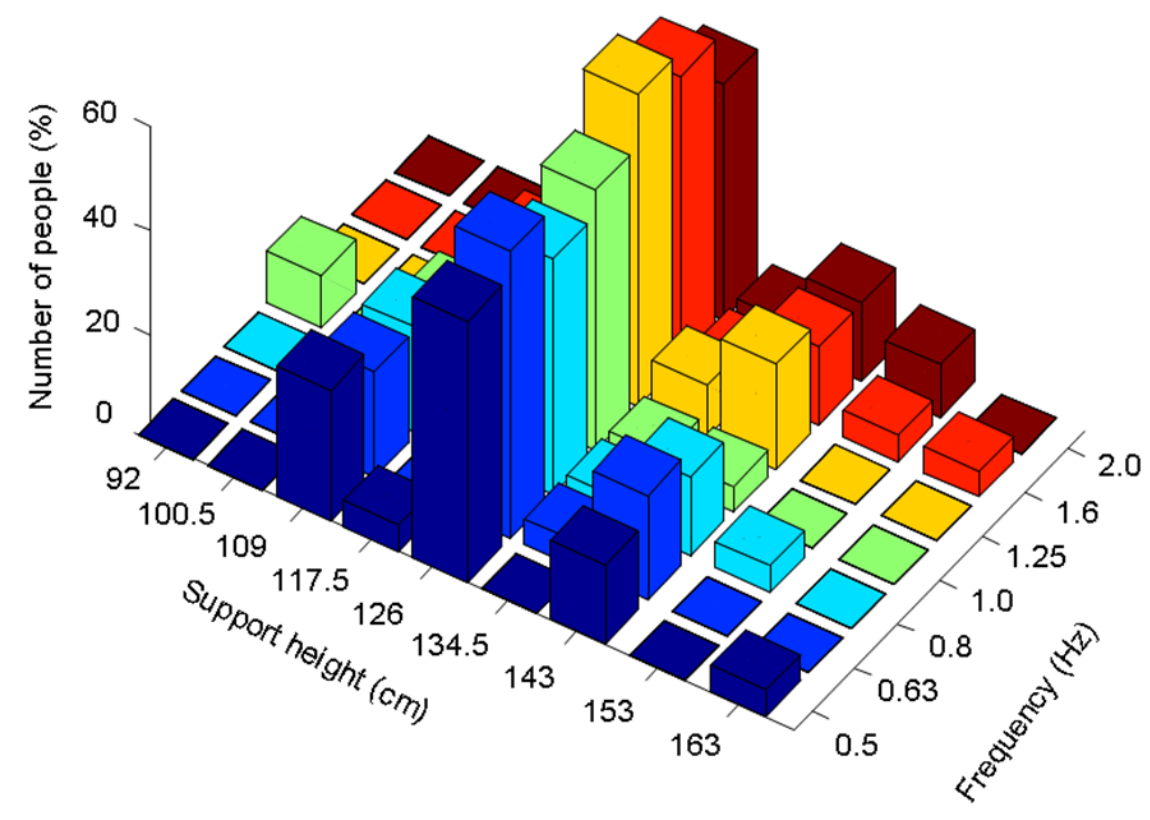

Fig. 6: Percentage of subjects preferring each support height at each frequency of oscillation with a velocity of $0.16 \mathrm{~ms}^{-1}$ r.m.s. 